\title{
T2c Stage Finding
}

National Cancer Institute

\section{Source}

National Cancer Institute. T2c Stage Finding. NCI Thesaurus. Code C48727.

A general term that refers to a TNM finding of a primary tumor growth beyond the level of in situ cancer, minimal subepithelial invasion, or minimal greatest diameter. The definition of T2 C TNM finding depends on the specific type of cancer that it refers to; for example, for prostate cancer it refers to a primary tumor that involves both lobes of the prostate gland; for ovarian cancer it refers to a primary tumor that involves one or both ovaries with extension or implants on the uterus and/or fallopian tubes, or other pelvic tissues, with malignant cells in either ascites or peritoneal washings; for fallopian tube cancer it refers to a primary tumor with pelvic extension and malignant cells in ascites or peritoneal washings. 